\title{
A Loaded Analysis Method for RV Cycloidal-pin Transmission Based on the Minimum Energy Principle
}

\author{
Tianxing $\mathrm{Li}^{1,2, *}$ - Hang $\mathrm{Xu}^{3}$ - Meng Tian ${ }^{2}$ \\ ${ }^{1}$ Henan University of Science and Technology, School of Mechatronics Engineering, China \\ ${ }^{2}$ Collaborative Innovation Center of Machinery Equipment Advanced Manufacturing of Henan Province, China \\ ${ }^{3}$ Zhongyuan University of Technology, School of Mechatronics Engineering, China
}

Due to the complexity of load distribution and contact conditions, as well as the lack of effective analysis methods, the theoretically designed rotary vector (RV) cycloidal-pin drive with good meshing characteristics shows poor loaded performance in practical applications. In this paper, an effective analysis method based on the minimum energy principle is proposed, which can accurately obtain the real loaded characteristics in line with the actual operations. In the process of loaded analysis, through the innovative introduction of the minimum energy principle, the actual number of teeth engaged simultaneously was accurately determined, which directly affects the quality of meshing. The results of simulation and measurement experiment demonstrate the correctness and practicability of the theoretical analysis method and the effectiveness of the introduction of the minimum energy principle. This study solves the problem that the actual meshing performance is inconsistent with the theoretical analysis results, and provides an effective way for the improvement and pre-control of the transmission accuracy and meshing quality of the robot $R V$ reducer.

Keywords: cycloidal-pin gear, loaded characteristics, minimum energy principle, rotary vector (RV) reducer, transmission error

\section{Highlights}

- An analysis method based on the minimum energy principle is proposed, which can accurately obtain the real loaded characteristics in line with the actual operations of the RV transmission.

- Through the innovative introduction of the minimum energy principle, the actual number of teeth engaged simultaneously is accurately determined.

- The possible meshing teeth and their numbers are quickly estimated according to the distribution of the minimum meshing clearance and the maximum deformation of the cycloidal-pin gear.

- The nonlinear mathematical relationship between the meshing load and the deformation is established.

\section{O INTRODUCTION}

The rotary vector (RV) reducer, accounting for about $38 \%$ of the total cost, is the core component of a robot [1] and [2]. The RV reducer is mainly composed of a two-stage mechanism. The cycloidal-pin gear at the low-speed stage is directly connected to the output shaft, so its transmission performance determines the output accuracy of a reducer [3] and [4], which directly affects the motion accuracy, repeated positioning accuracy and service life of the robot.

One of the main features of cycloidal-pin transmission is simultaneous multi-tooth engagement. The error equalization effect generated by multi-tooth meshing is conducive to improving the transmission accuracy, which allows high meshing rigidity and large carrying capacity. However, due to the complicated load distribution and contact conditions of the cycloidal-pin gear with simultaneous multitooth meshing, as well as the lack of effective load analysis methods, the theoretically designed RV mechanism with good meshing characteristics shows poor load performance in practical applications. Low transmission precision, insufficient bearing capacity, and short accuracy retention are common problems to be solved in RV transmission.

Many researchers have conducted in-depth studies on the meshing characteristics of cycloidalpin drives. Lin et al. [1] analysed the kinematic characteristics of the cycloidal drive by using the tooth contact analysis. Through analysing the contact dynamics in a cycloidal-pin transmission, $\mathrm{Xu}$ et al. [3] concluded that multi-tooth meshing characteristics have the main influence on the kinematic accuracy and the contact force. Based on unloaded and loaded contact analyses of the cycloid speed reducer, $\mathrm{Li}$ et al. [5] predicted the loads on various components in the presence of clearances and eccentricity errors. Li et al. [6] proposed an unloaded meshing analysis method for RV cycloidal-pin drive considering the influence of manufacturing errors. Based on the influence coefficient method, Huang and Tsai [7] proposed a computerized approach of loaded tooth contact analysis for the contact tooth pairs of the involute stage and the cycloid stage. Demenego et al. [8] developed a computer program for tooth contact analysis, which 
can determine the virtual pair of contacting profiles, the backlash and the transmission errors.

Furthermore, the research on the meshing characteristics of ordinary gears laid the foundation for cycloidal-pin transmission. Hao et al. [9] established an analysis model to obtain the loadsharing characteristics of the aeronautical two-stage five-branching planets gear train. Tsai and Ye [10] proposed a computerized approach based on the influence coefficient method to solve the statically indeterminate problem of multiple loaded contact tooth pairs in planetary gear drives. Wang et al. [11] solved the meshing characteristic problem of orthogonal face-gear drives in real working conditions. Bodzas [12] carried out loaded tooth contact analysis of straight bevel gears and obtained the mechanical characteristics by different loads. Vivet et al. [13] proposed a multi-body meshing analysis method and analysed the transmission error and contact patterns of spiral bevel gears. Lin [14] presented the loaded contact analysis of curve-face gear pair. Nishino [15] developed a computerized tool to predict the loaded tooth contact pattern and static transmission error of hypoid gears. Kolivand and Kahraman [16] proposed a practical methodology based on ease-off topography for loaded tooth contact analysis of hypoid gears. Batsch [17] conducted tooth contact analysis on the convexo-concave helical bevel Novikov gear. Wang et al. [18] improved the transmission characteristics of equal base bevel gears from a processing perspective. Yang et al. [19] proposed a dynamic analysis method to improve the transmission performance of spiral bevel and hypoid gears. Changbin et al. [20] simulated the dynamic meshing process of the elliptic cylinder gear.

The load distribution and actual contact situation of the multi-tooth meshing of the cycloidal-pin gear are extremely complicated, and its load-bearing transmission process is a multi-body contact process. The key to loaded analysis is to accurately determine the actual number of meshing teeth and the nonlinear relationship between the contact force and deformation. In order to solve the static problems of elastic components, the principle of minimum energy is often used. It avoids the complex process of integral calculation directly using the relationship between force and deformation but gives a more concise result from the stability of energy. Li et al. [21] proposed a calculation method for cutting force based on an energy method. Based on the energy balance, Wang et al. [22] predicted the contribution of the axial force of the gear. Wang and Feng [23] derived the formula of time-varying mesh stiffness of the gear train based on the energy method. Liang et al. [24] evaluated the time-varying mesh stiffness of a planetary gear set by means of the potential energy method. Wan et al. [25] proposed an accumulated integral potential energy method to calculate the mesh stiffness of helical gears. Song et al. [26] proposed a potential energy-based slice grouping method to calculate the mesh stiffness of straight bevel gears. Wang et al. [27] studied the chaos and stability of spur gear based on the energy method and floquet theory. The above work provides a novel idea for the study of the complex meshing characteristics of cycloidal-pin gears.

In this paper, the nonlinear mathematical relationship between the meshing load and the deformation is established first. Next, the possible meshing teeth and their numbers are quickly estimated according to the distribution of the minimum meshing clearance and the maximum elastic deformation. More importantly, the principle of minimum energy is innovatively introduced into the loaded analysis process to accurately determine the actual number of teeth engaged simultaneously and their tooth numbers. Finally, according to the loaded analysis model of cycloid-pin gear, some important parameters of loaded characteristics are calculated and analysed.

\section{TRANSMISSION CHARACTERISTICS OF CYCLOIDAL-PIN GEAR}

The RV reducer is a closed differential gear system composed of a two-stage transmission mechanism, as shown in Fig. 1. The sun gear (driving gear) is connected to the input shaft. The planet gear meshed with the sun gear is fixedly connected with the crankshaft. Two cycloidal gears with the phase difference of $180^{\circ}$ are installed on the crankshaft and engage with the pin gear fixed on the pin box. If the driving gear rotates counter-clockwise at a certain speed, the planetary gear meshing with the sun gear rotates clockwise, and the crankshaft fixed to the planetary gear rotates clockwise. The rotation of the crankshaft drives the cycloidal gear to revolve clockwise, and at the same time, the cycloidal gear rotates counter-clockwise under the action of the pin gear. The rotation of the cycloidal gear, in turn, causes the crankshaft to revolve counter-clockwise around the input shaft. The angular velocity of this revolution is the output angular velocity of the RV reducer, and its direction is the same as that of the input shaft. When the crankshaft rotates $360^{\circ}$, the cycloidal gear rotates by a pitch angle in the opposite direction. The rotation angular velocity of the cycloidal gear is equal to the revolution angular velocity of the crankshaft 
connected to the output mechanism, so the angular velocity of the cycloidal gear can be transmitted and output by the output mechanism at a speed ratio of $1: 1$. It can be seen that the transmission performance of the cycloidal-pin mechanism directly connected to the output shaft has an important effect on the motion accuracy of the RV reducer.

a)

First cycloidal gear Roller position
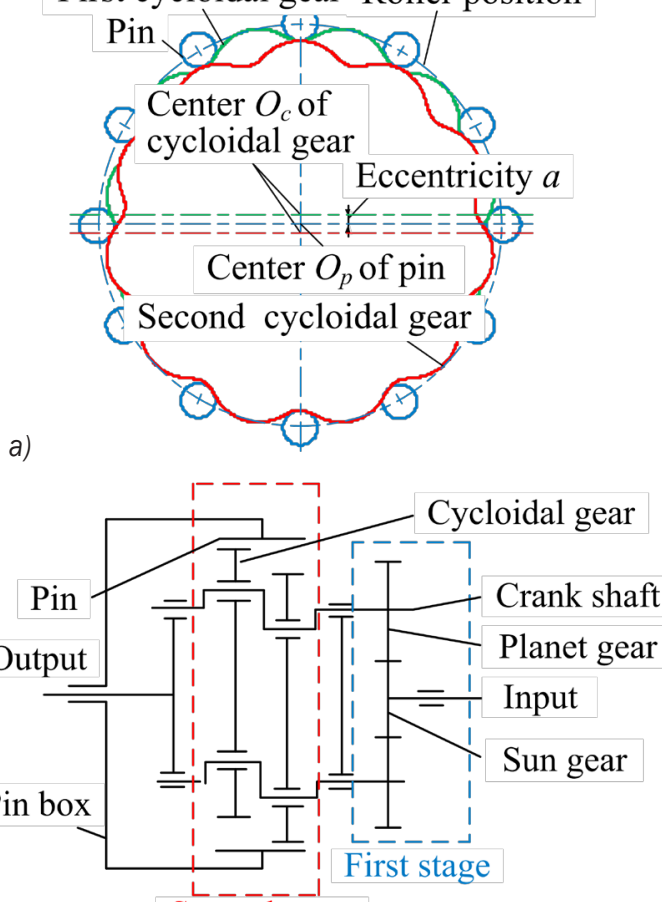

b)

Fig. 1. Transmission mechanism of $R V$ reducer; a) cycloidal-pin gear; and b) transmission mechanism

The simultaneous meshing of multiple teeth of the cycloidal-pin gear not only allows the load to be distributed to each tooth pair, which improves the carrying capacity, and its error equalization effect greatly improves the transmission accuracy. However, the load distribution and actual contact conditions of the multi-tooth meshing are quite complicated, and higher requirements are also placed on the machining errors and assembly processes [6]. In practical applications, many factors, such as machining errors, assembly errors, and modification errors, have significant and complex effects on the meshing contact state of gear teeth. In fact, according to the meshing principle of the gear, the interactive influence of various error factors indirectly changes the profile shape of the meshing tooth pair, which in turn affects the meshing contact state and transmission accuracy. In this case, the characteristics of simultaneous multi- tooth engagement no longer exist. Under no-load conditions, the smaller or larger cycloid gear and the pin gear only have a pair of teeth to maintain contact. There are initial clearances of different sizes between the other gear teeth. In the loaded state, only when the deformation of the first contact tooth is greater than the initial clearance, the remaining teeth can come into contact one after another. Therefore, the modification errors, machining errors, and thermal deformations have caused the change in the contact characteristics of cycloidal-pin gear, which inevitably produce transmission errors and lost motion. The change in the shape of the tooth profile leads to uneven load distribution, which will aggravate wear and reduce the accuracy retention of RV transmission.

\section{MESHING MODEL OF CYCLOIDAL-PIN GEAR}

Tooth contact analysis is one of the important means of studying meshing characteristics. The analysis method and model have been generated by some researchers [28] and [29]. In this section, the mathematical model of the cycloidal-pin transmission will be given for later use [6]. As shown in Fig. 2, $S_{f}\left(x_{f}, y_{f}\right)$ is a coordinate system fixedly connected to the frame. The coordinate systems of the pin gear and cycloidal gear are defined as $S_{p}\left(x_{p}, y_{p}\right)$ and $S_{c}\left(x_{c}, y_{c}\right)$, respectively. Their rotation angles are denoted as $\phi_{1}$ and $\phi_{2}$. The eccentricity is represented by $a$.

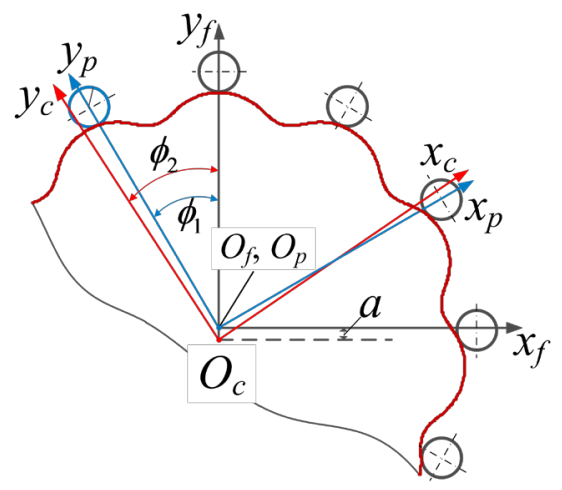

Fig. 2. Meshing contact model of cycloidal-pin gear

In the RV transmission process, the position vector and normal vector of the cycloidal gear and the pin gear at any meshing point should be consistent. Therefore, the meshing contact equation can be established [6]

$$
\left\{\begin{array}{l}
\mathbf{R}_{c}^{(f)}\left(\varphi, \phi_{2}\right)=\mathbf{R}_{p}^{(f)}\left(\beta, \phi_{1}\right) \\
\mathbf{n}_{c}^{(f)}\left(\varphi, \phi_{2}\right)=\mathbf{n}_{p}^{(f)}\left(\beta, \phi_{1}\right)
\end{array}\right.
$$


where the superscript $(f)$ is indicated in the coordinate system $S_{f}$. The vector $\mathbf{R}_{c}$ and $\mathbf{n}_{c}$ respectively represent the position and normal of the cycloidal gear, with $\varphi$ and $\phi_{2}$ as variables [1] and [6]. The vector $\mathbf{R}_{p}$ and $\mathbf{n}_{p}$ are the position and normal of the pin gear, with $\beta$ and $\phi_{1}$ as variables.

There are three nonlinear equations and four unknowns in Eq. (1). By giving different driving angle $\phi_{1}$, a series of driven angles $\phi_{2}$ and contact position parameters $\varphi$ and $\beta$ can be solved. The process of solving the meshing model is described as follows: first, an initial value of the driving angle $\phi_{1}$ is given, which is denoted as $\phi_{1}^{(0)}$. In this case, Eq. (1) is a nonlinear equation system consisting of three equations and three unknowns. The driven angle $\phi_{2}{ }^{(0)}$ and the initial contact position parameters $\beta^{(0)}$ and $\varphi^{(0)}$ can be solved by the optimization method. Then, by continuously changing $\phi_{1}$ in small increments, a series of meshing contact parameters $\phi_{1}^{(k)}, \phi_{2}^{(k)}, \beta^{(k)}$ and $\varphi^{(k)}$ can be solved (superscript $\mathrm{k}=1,2, \ldots$ is the index of the meshing contact point). The nonlinear relationship between $\phi_{1}$ and $\phi_{2}$ determines the transmission error. The parameters $\varphi$ and $\beta$ determine the contact position on a tooth profile. Under no-load conditions, the transmission error is mainly determined by the actual clearance between the gear teeth. In the loaded state, the transmission error is mainly caused by the contact deformation and the meshing clearance, which is the main difference between the meshing characteristics analysis under unloaded and loaded conditions.

\section{LOADED ANALYSIS BASED ON MINIMUM ENERGY PRINCIPLE}

In theory, the deformation quantity at a meshing position should be the sum of the contact deformation and bending deformation. However, in order to increase the rigidity of the mechanism, a pillowlike pin-tooth structure is often used. Therefore, the bending deformation is ignored, and the contact deformation is mainly considered in this paper. Also, the error equalization effect of multi-tooth engagement makes the two symmetrically installed cycloidal gears have similar meshing characteristics, so only one cycloidal gear is considered.

\subsection{Meshing Force at Contact Position}

In the loaded transmission, the cycloidal gear bears the load torque. At the same time, due to its meshing contact with the pin gear, a torque opposite to the direction of the load torque is also imposed. According to the principle of torque balance, when the RV transmission runs stably, the algebraic sum of all torques should be zero. Therefore, the nonlinear relationship between the torque and the meshing force can be established.

$$
T_{c}=\sum_{i=1}^{m} F_{i}\left(\varphi_{i}, \beta_{i}, \phi_{1 i}, \phi_{2 i}\right) \cdot L_{i}\left(\varphi_{i}\right),
$$

where $\mathrm{i}=1 \sim m, m$ is the number of simultaneous meshing teeth, and the meaning is the same below. The meshing force of the $i^{\text {th }}$ tooth is represented by $F_{i}$, which is related to parameters $\phi_{1}, \phi_{2}, \varphi$ and $\beta$. The distance $L_{i}$ is the force arm at the contact point of the $i^{\text {th }}$ tooth, with $\varphi$ as the variable.

By solving Eq. (1), the contact position parameters $\varphi$ and $\beta$ of the cycloidal gear and the pin gear can be determined. Assuming that the number of meshing teeth $m$ is determined, the force $\operatorname{arm} L_{i}$ can be calculated. According to Eq. (2), the meshing force of the contact position can be obtained.

\subsection{Meshing Contact Deformation and Coordination Relationship}

According to the Hertz contact theory, the elastic contact problem of the cycloidal-pin gear at the meshing position is simplified in this paper [30]. The contact is considered as the smooth contact of the elastic body without friction. The vertical pressure on the contact surface is considered to be uniformly distributed, and the contact area is a small deformation. Thus, the meshing contact of the cycloidal-pin gear can be simplified into an elastic contact between two parallel cylinders, as shown in Fig. 3.

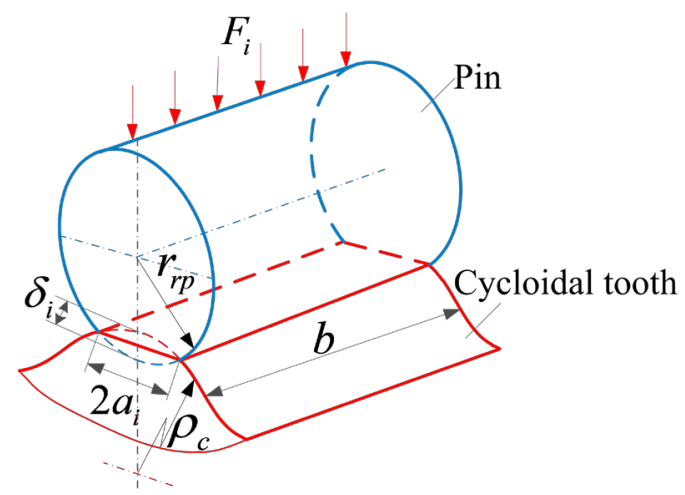

Fig. 3. Contact deformation model of cycloidal-pin gear

Under no-load conditions, the contact pattern of the cycloidal-pin gear should be a straight line. After loading, the contact pattern will become rectangular. The contact half-width $a_{i}$ can be expressed as: 


$$
\begin{aligned}
& a_{i}\left(\varphi_{i}, \beta_{i}, \phi_{1 i}, \phi_{2 i}\right)= \\
& \sqrt{\frac{4 \cdot F_{i}\left(\varphi_{i}, \beta_{i}, \phi_{1 i}, \phi_{2 i}\right) \cdot \rho_{i}\left(\varphi_{i}\right)}{\pi \cdot b}\left(\frac{1-\mu_{c}^{2}}{E_{c}}+\frac{1-\mu_{p}^{2}}{E_{p}}\right)},
\end{aligned}
$$

where $b$ is the width of the cycloidal gear. Young's modulus of the cycloidal gear and the pin gear are denoted as $E_{c}$ and $E_{p}$, respectively. Their Poisson ratio are $\mu_{c}$ and $\mu_{p}$. The comprehensive curvature radius, $\rho_{i}$, at the meshing point, which can be determined by the radius of curvature of the cycloidal gear and the pin gear.

$$
\rho_{i}(\varphi)=\frac{1}{\frac{1}{\rho_{c i}(\varphi)} \pm \frac{1}{r_{r p}}}=\frac{\rho_{c i}(\varphi) \cdot r_{r p}}{\rho_{c i}(\varphi) \pm r_{r p}},
$$

where the positive sign is used for external contact and the negative sign is used for internal contact. The roller radius, which is represented by $r_{r p}$, is the radius of curvature of the pin gear. The radius of curvature at the contact point of the cycloidal gear is denoted as $\rho_{c i}$, which can be calculated as follows:

$$
\rho_{c i}(\varphi)=\left|\begin{array}{l}
\left(r_{p}+\Delta r_{p}\right) \cdot \\
\frac{\left(1+k_{1}^{2}-2 k_{1} \cdot \cos \left(z_{c} \cdot \varphi\right)\right)^{3 / 2}}{k_{1} \cdot\left(z_{p}+1\right) \cdot \cos \left(z_{c} \cdot \varphi\right)-\left(1+z_{p} \cdot k_{1}^{2}\right)} \\
r_{r p}+\Delta r_{r p}
\end{array}\right|,
$$

where $z_{c}$ and $z_{p}$ is the number of teeth of cycloidal gear and pins; $k_{1}$ is the short width coefficient; $r_{p}$ is the roller position; $\Delta r_{p}$ and $\Delta r_{r p}$ is the modification by the radius and position of the roller.

According to the geometric relationship in Fig. 3, the deformation $\delta_{c i}$ and $\delta_{p i}$ of the cycloidal-pin gear at the $i^{\text {th }}$ contact position can be calculated as follows

$$
\begin{gathered}
\delta_{c i}=\rho_{c i}-\sqrt{\rho_{c i}^{2}-a_{i}^{2}}, \\
\delta_{p i}=r_{r p}-\sqrt{r_{r p}^{2}-a_{i}^{2}} .
\end{gathered}
$$

The normal stiffness $K_{c i}$ and $K_{p i}$ at the $i^{\text {th }}$ contact position of the cycloidal gear and the pin gear can be determined respectively:

$$
\begin{aligned}
& K_{c i}=\frac{F_{i}}{\delta_{c i}}, \\
& K_{p i}=\frac{F_{i}}{\delta_{p i}} .
\end{aligned}
$$

From this, the normal meshing stiffness $K_{n i}$ of a pair of teeth of the cycloid-pin gear can be obtained:

$$
K_{n i}=\frac{K_{c i} K_{p i}}{K_{c i}+K_{p i}} .
$$

The equivalent torsional stiffness $K_{t i}$ of the cycloidal-pin gear at the $i^{\text {th }}$ contact position can be expressed as follows:

$$
K_{t i}=K_{n i} \cdot L_{i}^{2} .
$$

Therefore, the nonlinear relationship between the meshing force $F_{i}$ and the deformation $\delta_{i}$ can be determined as follows:

$$
\delta_{i}\left(\varphi_{i}, \beta_{i}, \phi_{1 i}, \phi_{2 i}\right)=\frac{F_{i}}{K_{t i}} .
$$

Additionally, the contact deformation of the cycloidal gear and the pin gear is not independent. A certain coordination relationship should be met, which ensures the integrity and continuity after contact deformation. In the paper, the ratio of the sum of $\delta_{i}$ and $d_{i}$ to $L_{i}$ is defined as the error rotation angle. According to the coordination relationship, the angles should be equal at all contact positions. Thus, the deformation coordination equation can be obtained

$$
\frac{\delta_{1}+d_{1}}{L_{1}}=\frac{\delta_{2}+d_{2}}{L_{2}}=\cdots=\frac{\delta_{i}+d_{i}}{L_{i}}=\cdots=\frac{\delta_{m}+d_{m}}{L_{m}} .
$$

\subsection{Determination of Possible Number of Meshing Teeth}

Under no-load conditions, only one pin tooth maintains meshing contact with the cycloidal tooth, and there are initial clearances of different sizes between the other teeth. According to Eq. (1), the distribution of the minimum meshing clearance of the cycloidal-pin gear can be obtained [6], as shown in Fig. 4.

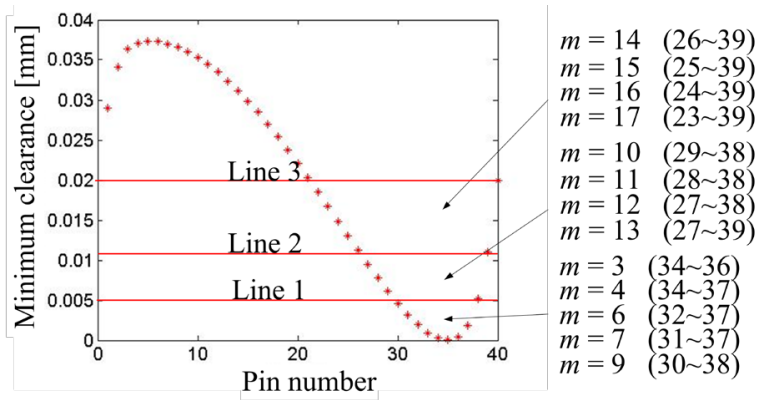

Fig. 4. Minimum meshing clearance and maximum deformation

It can be seen from the distribution of the minimum clearance shown in Fig. 4 that the clearance between the $35^{\text {th }}$ pin tooth and the cycloidal profile is 
zero. Therefore, the $35^{\text {th }}$ pin should be the first tooth in contact under no-load condition. After loading, the $35^{\text {th }}$ pin tooth produces a certain deformation displacement under the action of the meshing force. When the next pin comes into contact, the deformation quantity reaches the maximum value. Only when the deformation value is greater than the meshing clearance of other pin teeth can they come into contact with the cycloidal tooth. Lines 1, 2, and 3 in Fig. 4 illustrate the position of the maximum deformation with respect to the clearance distribution. If the maximum deformation is at the position of line 1, the No. 30 38 pin teeth bellow line 1 will be most likely to contact after load. In this case, the maximum number of meshing teeth can be estimated to be 9 .

It should be noted that the estimated number of meshing teeth and their tooth numbers are only the maximum possible value. Each possibility needs to be grouped to discuss the actual number of meshing teeth. The detailed process of estimating the number of teeth that may be meshed and their tooth numbers is shown in Fig. 5.

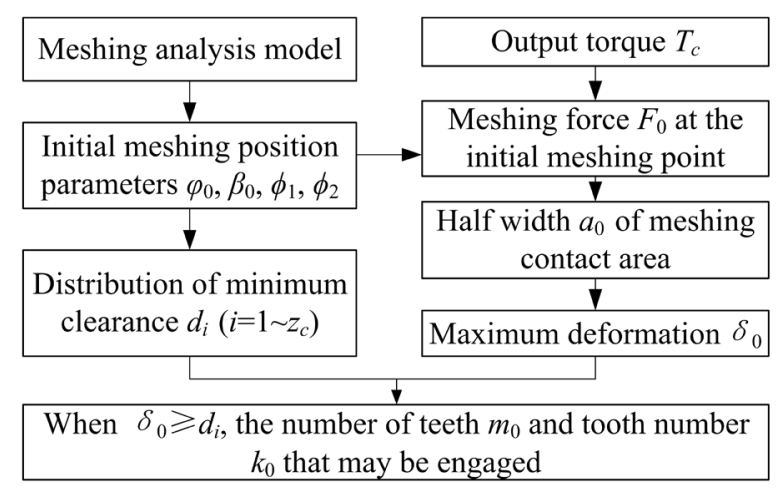

Fig. 5. Flowchart of determination of the possible number of meshing teeth

\subsection{Actual Number of Meshing Teeth Based on Minimum Energy Principle}

Energy is conserved. In order to maintain the balance of the system, the energy will automatically decrease until the whole system reaches a state of dynamic balance. The state at the minimum energy is relatively stable. The RV cycloidal-pin transmission should also conform to this energy conversion process.

The total energy of the cycloidal-pin transmission system in the stable state should be the minimum. Therefore, the principle of minimum energy can be used to accurately determine the actual number of meshing teeth of the cycloidal-pin gear. The total energy $N E$ of the cycloidal-pin gear is the sum of the product of the meshing force $F_{i}$ and the deformation $\delta_{i}$, which can be expressed as follows:

$$
\begin{aligned}
& N E\left(\varphi_{i}, \beta_{i}, \phi_{1 i}, \phi_{2 i}\right)= \\
& \sum_{i=1}^{m} F_{i}\left(\varphi_{i}, \beta_{i}, \phi_{1 i}, \phi_{2 i}\right) \cdot \delta_{i}\left(\varphi_{i}, \beta_{i}, \phi_{1 i}, \phi_{2 i}\right),
\end{aligned}
$$

The specific method is to first group the possible meshing tooth pairs of the cycloidal-pin gear under the loaded state, and then calculate and compare the total energy of each group separately. The number of meshing teeth with the minimum total energy is the actual number of meshing teeth. The actual meshing state of the cycloidal-pin gear can be determined based on the number of teeth engaged simultaneously and their tooth numbers. The determination process is shown in Fig. 6.

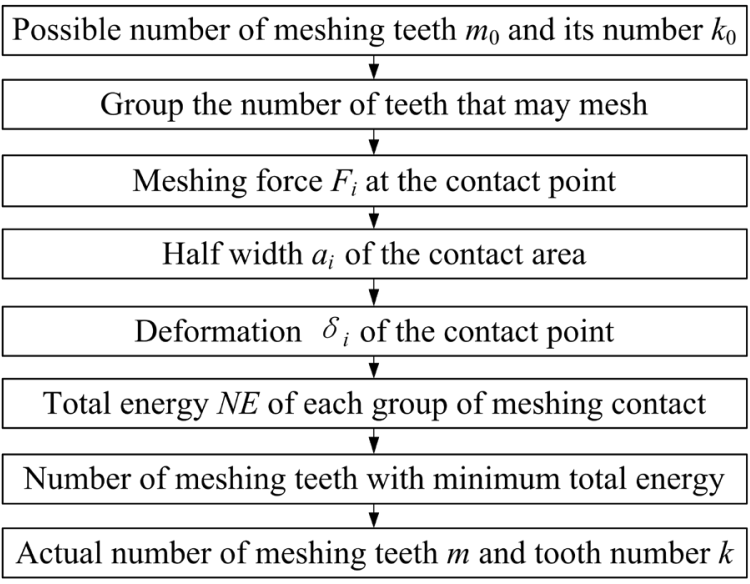

Fig. 6. Flowchart of determination of the actual number of meshing teeth

\subsection{Loaded Analysis Strategy}

According to the loaded analysis model and calculation method of meshing parameters, the loaded analysis process of the cycloidal-pin gear is planned, as shown in Fig. 7. The specific implementation process is as follows:

1. According to the unloaded contact analysis method, the initial meshing position of the cycloidal-pin gear is solved. On this basis, the distribution of the minimum meshing clearance of the RV transmission is determined.

2. According to the loaded analysis model, the maximum deformation at the initial meshing position is determined.

3. By comparing the minimum meshing clearance and the maximum deformation, the possible number of meshing teeth and the tooth numbers 
are estimated, and the reasonable grouping is performed.

4. Based on the loaded analysis model, the meshing force and deformation at the meshing contact point of each group are calculated in sequence, and then the total energy of deformation of each group is obtained.

5. By comparing the total energy of each group, the meshing force, deformation, the number of simultaneous meshing teeth and the tooth numbers, as well as the meshing position parameters, are determined at the minimum energy. These data reflect the actual loaded meshing state.

6. Continuously changing the rotation angle of driving gear and the contact position, the instantaneous meshing parameters of the corresponding meshing point can be calculated in sequence until it is disengaged.

7. According to the meshing position parameters, the loaded contact patterns can be determined. According to the relationship between the theoretical and actual output angle, the loaded transmission error curve can be drawn.

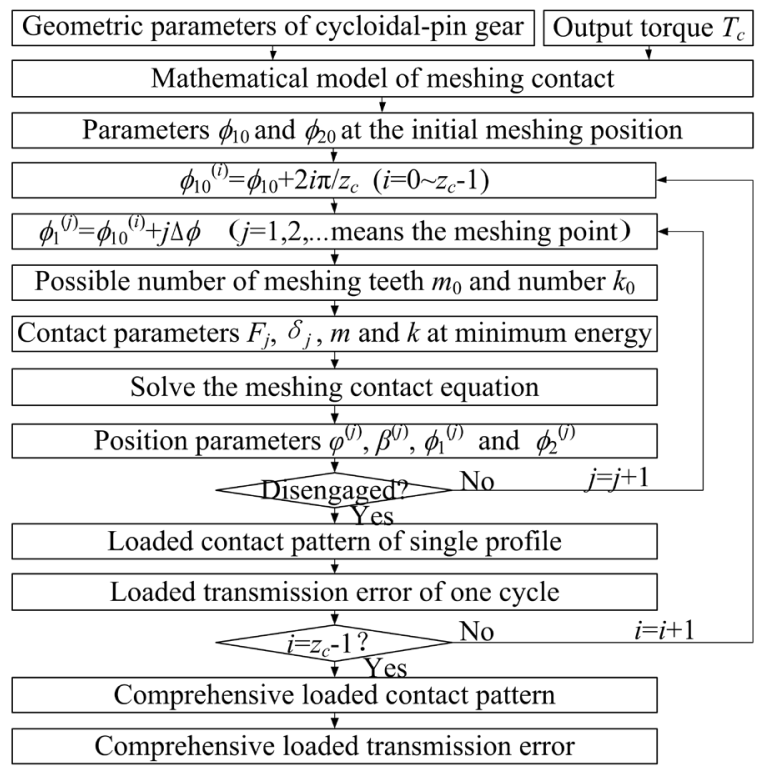

Fig. 7. Flowchart of loaded analysis

\section{RESULTS OF LOADED ANALYSIS}

Taking the cycloidal-pin gear of a robot reducer as an example, the loaded meshing performance is analysed according to the proposed strategy. The cycloidal gear and the pin gear are made of GCr15 bearing steel with the Poisson ratio of 0.3 and Young's modulus of $2.08 \times 10^{5} \mathrm{MPa}$. The geometric parameters of the cycloidal-pin gear are shown in Table 1.

In the experiment, $10 \%$ of the rated torque, $T_{c}=57 \mathrm{~N} \cdot \mathrm{m}$, was applied to the cycloidal-pin gear. According to the loaded analysis model, the meshing force and deformation energy of the cycloidal-pin gear were solved. The actual number of meshing teeth is $m=16$ and the tooth number $k$ is No. $24 \sim 39$. According to the planned strategy of loaded analysis, the deformation, meshing stress, contact patterns and loaded transmission errors at each meshing point were obtained.

Table 1. Geometric parameters of cycloidal-pin gear

\begin{tabular}{lc}
\hline Parameters & Values \\
\hline Number of teeth of sun gear & 12 \\
\hline Number of teeth of planet gear & 48 \\
\hline Pressure angle [deg] & 20 \\
\hline Number of pins & 40 \\
\hline Number of teeth of cycloid gear & 39 \\
\hline Roller position [mm] & 52.15 \\
\hline Roller radius [mm] & 2 \\
\hline Eccentricity [mm] & 0.9 \\
\hline Width of cycloidal gear [mm] & 10 \\
\hline Width of pin gear [mm] & 22 \\
\hline Modification by roller radius [mm] & 0.01 \\
\hline Modification by roller position [mm] & -0.012 \\
\hline
\end{tabular}

\subsection{Loaded Contact Force and Contact Pressure}

Fig. 8 shows the contact force and its distribution at each meshing point when the rotation angle of the pin gear is $0.02 \mathrm{rad}, 0.04 \mathrm{rad}, 0.06 \mathrm{rad}$, and $0.08 \mathrm{rad}$ respectively.

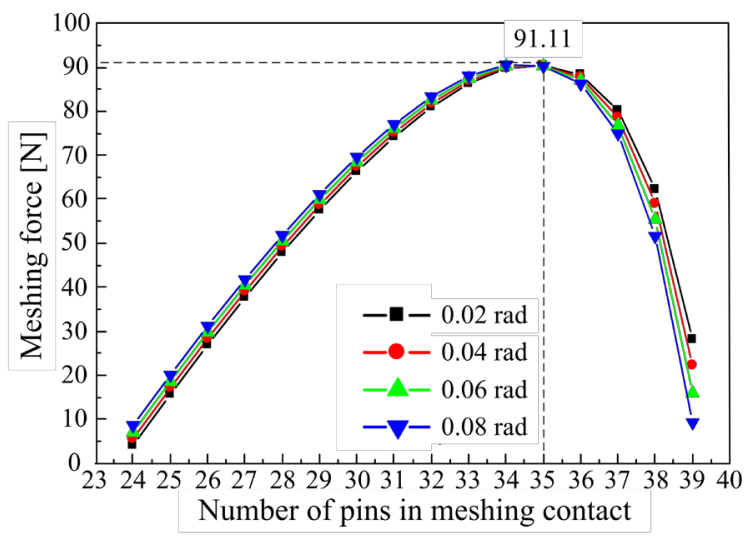

Fig. 8. Contact force distribution at meshing points

It can be seen from Fig. 8 that the force distribution of the meshing positions at different 
rotation angles of the cycloidal-pin gear is essentially the same. As the number of pins in meshing contact gradually increases, the contact force first increases to the peak and then decreases. The maximum contact force is $91.11 \mathrm{~N}$ at the $35^{\text {th }}$ pin. If the pin gear rotates counter-clockwise, as the rotation angle of the pin gear increases, the contact force before the peak increases, and the contact force after the peak decreases. According to the distribution of the contact force, with the rotation of the pin gear, the contact force of the $39^{\text {th }}$ pin will gradually decrease until it reaches 0 , at which time this pair of gear teeth are disengaged. At the same time, the $23^{\text {rd }}$ pin teeth come into contact in sequence, and the contact force increases until it reaches the peak and then gradually decreases until disengaged.

According to the contact force and contact area at each meshing position, the contact pressure was also calculated, as shown in Fig. 9.

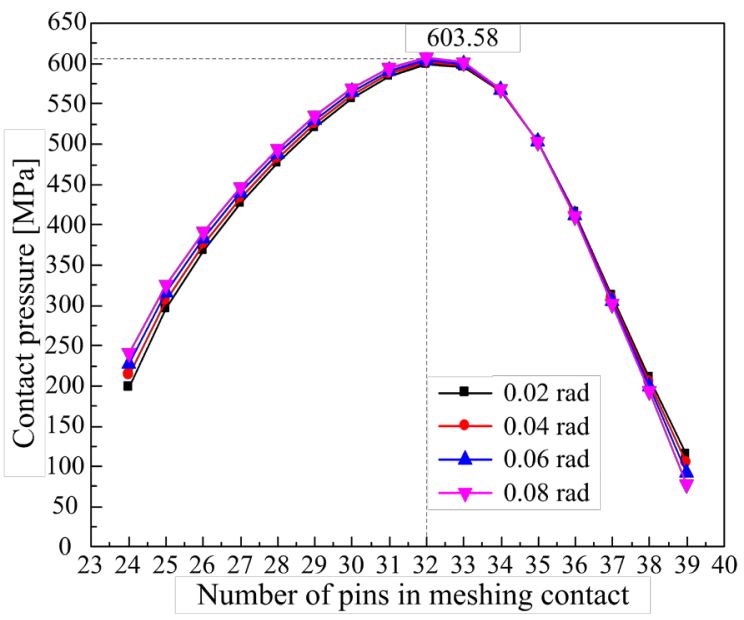

Fig. 9. Contact pressure at meshing points

It can be seen that the contact pressure at each meshing position increases to the peak first and then decreases with the increase of the number of pins. The maximum pressure is at the $32^{\text {nd }}$ pin tooth, and its value is $603.58 \mathrm{MPa}$. Compared with the distribution of the contact force in Fig. 8, the peak position of the maximum contact pressure is different. The pressure value is related not only to the contact force $F_{i}$, but also to the comprehensive curvature radius $\rho_{i}$. If the contact force is larger, the corresponding comprehensive radius of curvature is also larger, but the contact pressure is not necessarily larger. Therefore, the influence of the curvature radius on contact pressure is more obvious.

\subsection{Loaded Deformation}

Fig. 10 shows the deformation distribution at each meshing point when the rotation angle of the pin gear is different.

It can be seen from Fig. 10 that the loaded deformation at the meshing position of the cycloidalpin gear is essentially consistent with the contact force. With the increase of the pin number, the deformation value at each meshing point first increases to the peak and then decreases. The maximum deformation is $0.057 \mathrm{~mm}$ at the $35^{\text {th }}$ pin. If the pin gear rotates counter-clockwise, the deformation before the peak increases, and the deformation after the peak decreases with the increase of the angle of the pin gear. According to the deformation distribution trend, with the rotation of the pin gear, the deformation of the $39^{\text {th }}$ pin gradually decreases until it becomes zero, at which time the pair of pin teeth are disengaged. At the same time, the 23 rd pin tooth is in meshing contact immediately, and its deformation quantity increases until it reaches the peak and then gradually decreases until disengaged.

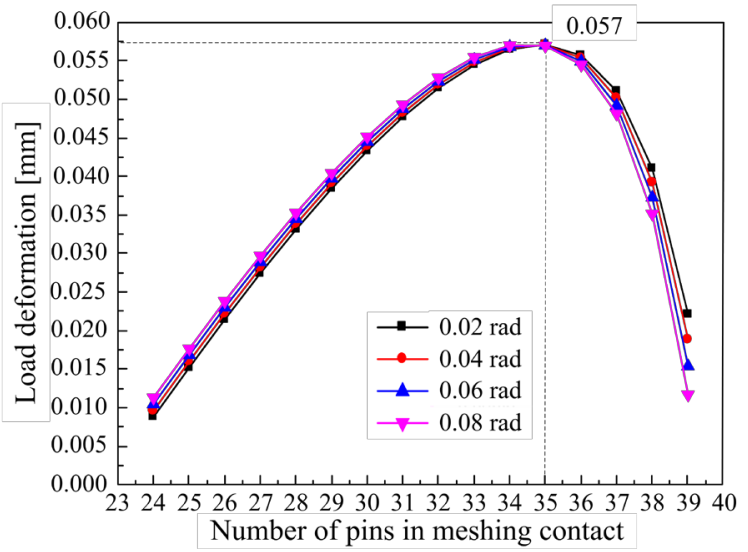

Fig. 10. Deformation distribution at each meshing point

According to Eq. (12), the deformation $\delta_{i}$ is influenced by the contact force $F_{i}$ and the curvature radius $\rho_{i}$. The changes in the meshing force and deformation shown in Figs. 8 and 10 are similar. It can be concluded that the curvature radius $\rho_{i}$ has little effect on the loaded deformation.

\subsection{Loaded Contact Pattern}

At the initial meshing position of the cycloidal-pin gear, the contact parameter $\varphi_{i}$ and the half-width $a_{i}$ were calculated, as shown in Table 2 . 
Table 2. Position parameters and contact half-width

\begin{tabular}{lccccccccc}
\hline Parameters & \multicolumn{7}{c}{ Number of simultaneous meshing teeth } \\
\hline Pin number & 24 & 25 & 26 & 27 & $\ldots$ & 36 & 37 & 38 & 39 \\
\hline Position [rad] & -2.51 & -2.36 & -2.2 & -2.04 & $\ldots$ & -0.63 & -0.47 & -0.31 & -0.16 \\
\hline Half-width [mm] & 0.15 & 0.19 & 0.24 & 0.27 & $\ldots$ & 0.44 & 0.37 & 0.34 & 0.24 \\
\hline
\end{tabular}

In order to more clearly compare the positions of the contact patterns when multiple teeth are in contact, all positions are displayed on the same tooth surface, as shown in Fig. 11. It can be seen that the loaded contact pattern of the cycloidal-pin gear is approximately rectangular. From the $24^{\text {th }}$ to $39^{\text {th }}$ teeth, the contact patterns at each meshing position are gradually widened from $0.15 \mathrm{~mm}$ to $0.47 \mathrm{~mm}$, and then gradually reduced to $0.24 \mathrm{~mm}$. The change rule of the loaded contact pattern is essentially the same as that of the meshing force and contact pressure, that is, the greater the loaded contact force and the contact pressure is, the larger the area of the contact pattern is.

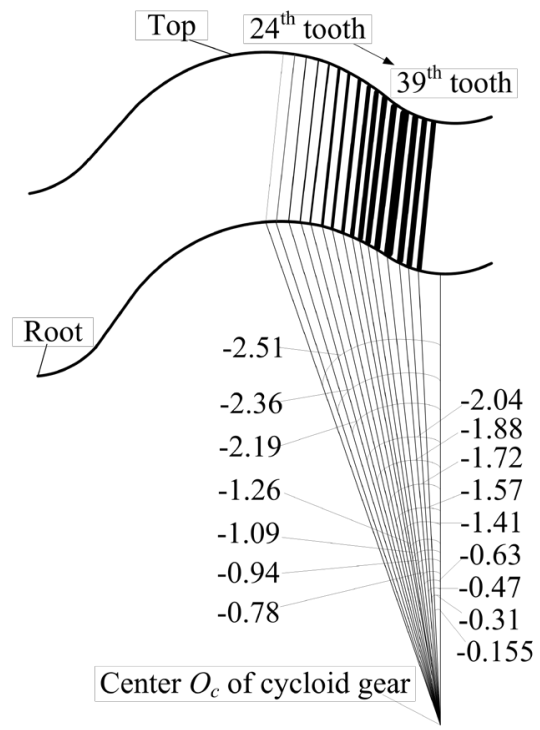

Fig. 11. Loaded contact patterns

\subsection{Loaded Transmission Error}

Using the loaded analysis model, continuously changing the rotation angle $\phi_{1}$ of the pin gear, the actual rotation angles of the cycloidal gear can be obtained. Then the loaded transmission error curve was drawn, as shown in Fig. 12.

It can be seen from Fig. 12 that the loaded transmission error of the cycloidal-pin gear changes periodically and is smooth. In one cycle, as the rotation angle of the pin gear increases, the transmission error gradually decreases to -3.80 arcmin and then slowly increases to -7.46 arcmin until the next cycle begins. The amplitude of the fluctuation is approximately 3.66 arcmin. Since the assembly error and machining error are not considered, the transmission error in the theoretical model mainly depends on the contact deformation, torsional deformation and meshing clearance. Therefore, the fluctuation amplitude of each meshing cycle is consistent and relatively small.

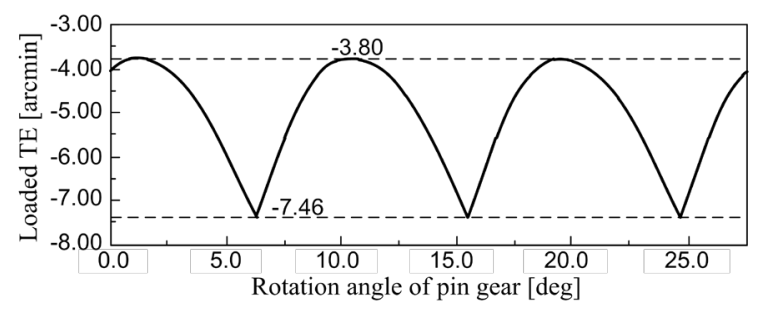

Fig. 12. Loaded transmission error curve (Three cycles)

It should be mentioned that the design purpose of the holes in the blank of the cycloid is mainly to reduce the weight. The prerequisite for lightweight design is that the meshing performance of the gear train cannot be changed. Therefore, the effect of the stiffness of holes in the blank on the contact pressure and transmission error can be ignored, which was proved in the comparison and analysis of ABAQUS results.

\section{EXAMPLES AND DISCUSSION}

The parametric modelling of the RV reducer was completed through Unigraphics (UG) software. Due to the pillow-like structure of the pin gear and the symmetrical distribution of the cycloidal gear in the robot reducer, the model is simplified to the meshing contact form of a cycloidal gear and a pin gear. The simplified model was imported into ABAQUS for finite element analysis.

In order to better compare the examples, two ABAQUS models have been established, as shown in Fig. 13. Model A in Fig. 13a is the "full blank". Model B in Fig. 13b is the "blank with holes".

The finite element mesh has a significant impact on the analysis results. The meshing contact 
deformation of the cycloidal-pin gear is mainly located near the contact area. Therefore, a method of mesh refinement near the contact area was adopted. The approximate size of the elements of the refined mesh is $0.2 \mathrm{~mm}$. The element type is C3D8R (8-node linear brick). The total number of meshes of the cycloidal gear (without holes) and the pin gear are 203,973 and 183,792 , respectively. Also, the gear axes (Z-axis) of the cycloid and pin gears were free, and other degrees of freedom were restricted. The bending moment imposed on a cycloidal gear was set to $28.5 \mathrm{Nm}$, which is half of the actual load. The contact property was defined as frictionless and "hard" contact. The pin surface was assigned as the master surface and the cycloidal surface as the slave surface.

a)

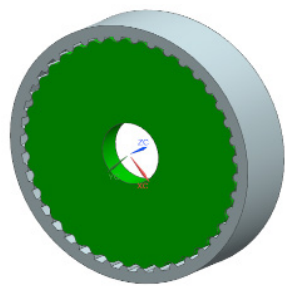

Fig. 13. ABAQUS model; a) model $A$; and b) model $B$

\subsection{Comparison and Analysis of Contact Pressure}

The contact pressure distributions of the finite element simulation of the cycloidal gear and the pin gear are shown in Figs. 14 and 15, respectively. It can be seen from Fig. 14 that the maximum contact pressures of the cycloidal gear are $601.3 \mathrm{MPa}$ and $600.0 \mathrm{MPa}$, respectively. The difference between the two is within $0.22 \%$, which shows that the stiffness of the hole in the blank has almost no effect on the contact pressure. For the pin gear in Fig. 15, the conclusions are similar.

a)

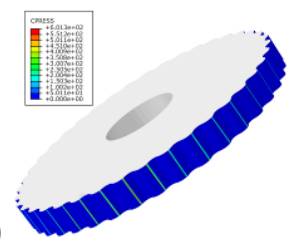

Fig. 14. Contact pressure distribution of the cycloidal gear; a) Model A; and b) Model B

In order to verify the correctness of the theoretical model, the contact pressure distribution of the Model B simulation and the theoretical calculation was compared in Fig. 16. It can be seen that their trends are similar, and the maximum pressure is at the $32^{\text {nd }}$ pin. The difference between the two is within $0.6 \%$,

which shows the correctness of the theoretical model and the results.

a)

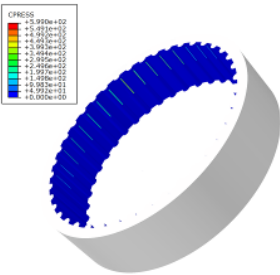

Fig. 15. Contact stress distribution of the pin gear; a) Model A; and b) Model B

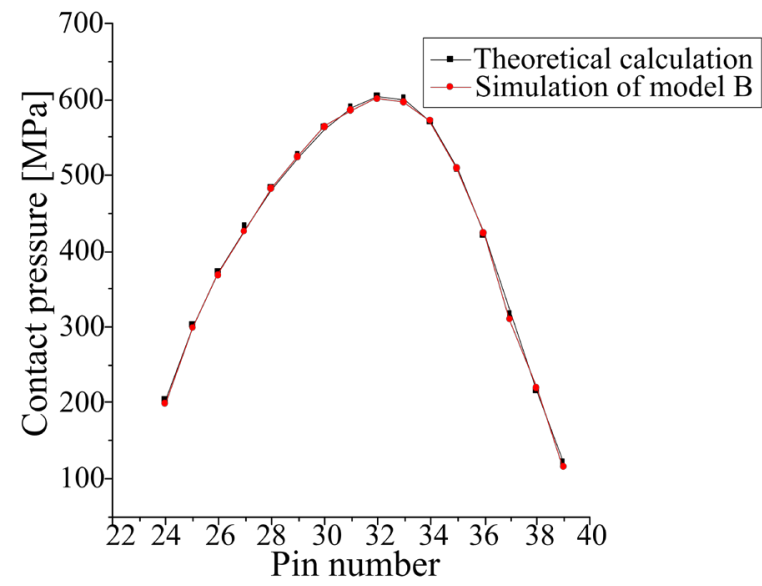

Fig. 16. Comparison of contact pressure between simulation and theoretical calculation

\subsection{Comparison and Analysis of Contact Pattern}

According to the contact stress of the finite element analysis, the actual number of meshing teeth and the tooth numbers were determined, and the meshing patterns of the cycloidal-pin gear was obtained, as shown in Fig. 17.

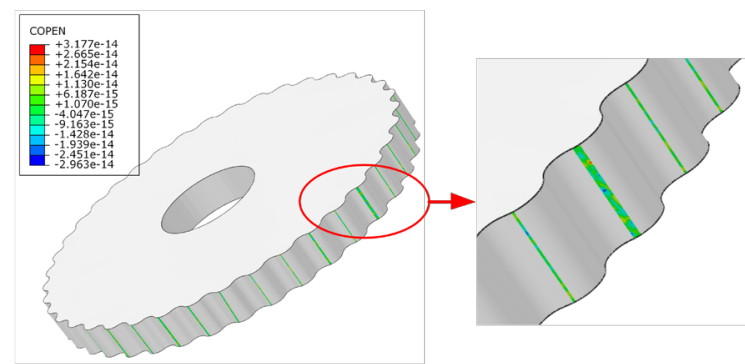

Fig. 17. Contact patterns of simulation

From the simulation results, it was found that the contact patterns of Models A and B were the same, which shows that the stiffness of the holes in the cycloidal gear has a very small effect on the contact 
patterns. Only the results of Model A were drawn in Fig. 17. It can be seen from the comparison with Fig. 11 that the actual number of meshing teeth is also 16 . The size of the meshing area is slightly different from the theoretical one, which is due to the influence of the mesh fineness in the simulation model and the rotation angle error of the theoretical model on the comparison results.

\subsection{Measurement of Loaded Transmission Error}

In order to better compare and analyse the results of the theoretical model and the ABAQUS model, the loaded transmission errors of Models A and B in ABAQUS were respectively extracted, as shown in Fig. 18.

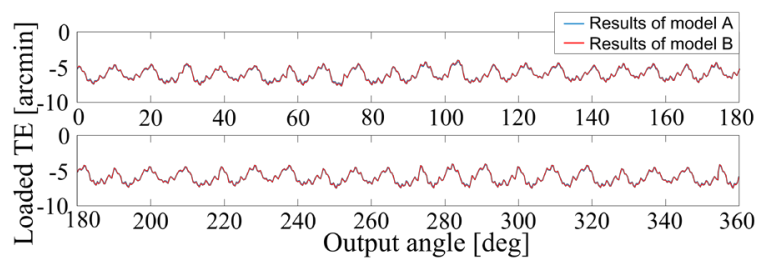

Fig. 18. Loaded transmission errors of Models $A$ and $B$

In Fig. 18, the fluctuation amplitudes of loaded transmission errors of models $\mathrm{A}$ and $\mathrm{B}$ are 3.69 arcmin and 3.7 arcmin, respectively. The difference in simulation results is 0.01 arcmin. Therefore, the influence of the stiffness of the holes in Model B on the loaded transmission error can be ignored. The simulation results of Models A and B are the same. In addition, compared with the theoretical results in Fig. 12, the difference between Model B and the theoretical model is within $1.1 \%$, which indicates the correctness of the theoretical model.

Furthermore, a measurement experiment of the loaded transmission error was completed on a RV reducer comprehensive performance tester, as shown in Fig. 19. The assembled reducer and its cycloidal gear with holes are shown in Fig. 20. During the measurement process, the RV reducer is mounted on a precision bracket. The input is driven by the input motor. The output end is connected to the output assembly through a coupling, and is loaded through a load motor and a torque sensor. Through the highprecision circular grating installed at the input and output ends, the angle changes of the input and output are collected in real time to realize the transmission error measurement. The measurement accuracy of the tester is within 8.0 arcsec, which can meet the experiment requirements.

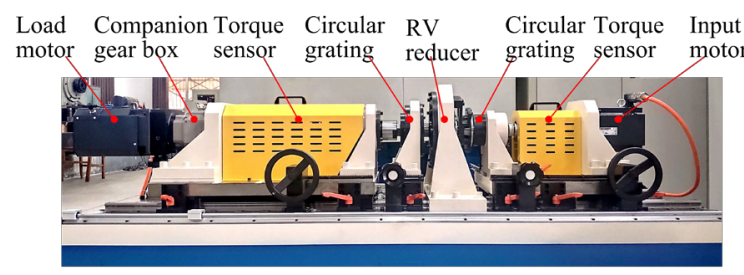

Fig. 19. RV reducer comprehensive performance tester
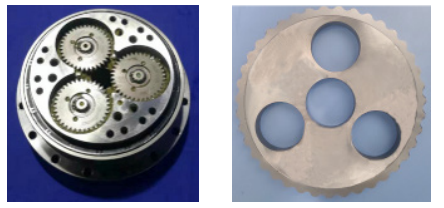

Fig. 20. a) $R V$ reducer and b) cycloidal gear

In the $360^{\circ}$ range of the output shaft, 1800 angles of the output shaft were collected. By calculating the difference between the theoretical and actual output angle, the loaded transmission error curve was drawn, as shown in Fig. 21.

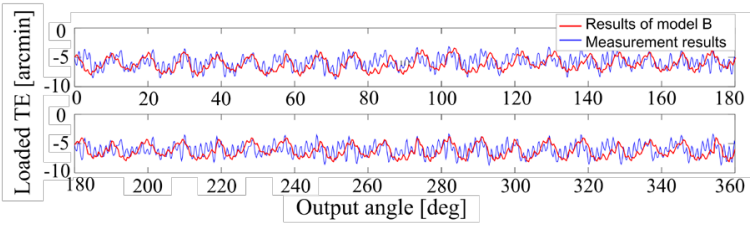

Fig. 21. Loaded transmission errors of measurement

It can be seen from Figs. 12 and 21 that the transmission error curves of theoretical calculation, actual measurement and simulation analysis all show a relatively consistent parabolic trend. In Fig. 21 , the actual measured transmission error is larger than those of the numerical model and experimental results. Their maximum fluctuation amplitudes are 3.83 arcmin, 3.66 arcmin and 3.7 arcmin, respectively. The maximum difference is about $4.4 \%$. From the comparison of data and trends, it can be concluded that the transmission error of the numerical model, the finite element model and the experimental results have good consistency. The effectiveness and practicability of the proposed method and simulation analysis can also be validated.

However, further improvement of the experimental results is an important topic for future work. The second-stage mechanism in the RV reducer has a certain influence on the experimental results. In addition, there are many influencing factors in the actual measurement process, such as assembly errors, profile errors and pitch errors of cycloidal gears, processing errors of other key components, bearing clearance, etc. The comprehensive influence of these 
factors is very complex and cannot be accurately determined [31] to [33].

\section{CONCLUSIONS}

This paper presents the loaded analysis method based on the minimum energy principle for RV cycloidal-pin transmission. The main conclusions include:

1. The Hertz contact theory, the principle of minimum energy and the finite element method were integrated into the loaded contact analysis of the cycloidal-pin drive, and the actual loaded characteristics were obtained.

2. The possible meshing teeth and their numbers were quickly estimated according to the distribution of the minimum meshing clearance and the maximum deformation of the cycloidalpin gear.

3. By comparing the minimum energy of the contact tooth pair, the actual number of teeth engaged simultaneously was accurately determined, which is critical to the accuracy of the loaded analysis.

4. The comparison and verification of theoretical modelling, finite element analysis and measurement experiment show that the difference in contact pressure, the number of meshing teeth, contact pattern and transmission errors were essentially the same, which proves the effectiveness and correctness of the proposed method.

This study solves the bottleneck problem in which the actual meshing performance is inconsistent with the theoretical analysis results in the loaded transmission of RV reducer, and has an important reference value for the improvement and pre-control of the kinematic accuracy of the robot RV Reducer. However, for the proposed method, some work remains to be done, including the comprehensive transmission characteristics of $\mathrm{RV}$ reducer in the theoretical model, the influence law of load torque on transmission error and the number of meshing teeth, and experimental research more in line with the actual working conditions.

\section{ACKNOWLEDGEMENTS}

This work supported by National Natural Science Foundation of China (No. U1504522, 51705134, and 51675161), Key Scientific Research Projects of Higher Education Institutions in Henan Province of China (No. 20A460010) and Key Scientific and Technological Project in the Henan Province of China (No. 202102210079).

\section{REFERENCES}

[1] Lin, W.S., Shih, Y.P., Lee, J.J. (2014). Design of a two-stage cycloidal gear reducer with tooth modifications. Mechanism and Machine Theory, vol. 79, p. 184-197, D0l:10.1016/j. mechmachtheory.2014.04.009.

[2] Li, T.X., Zhou, J.X., Deng, X.Z., Li, J.B., Xing, C.R., Su, J.X., Wang, H.L. (2018). A manufacturing error measurement methodology for a rotary vector reducer cycloidal gear based on a gear measuring center. Measurement Science and Technology, vol. 29, art. ID 075006, D0I:10.1088/1361-6501/aac00a.

[3] Xu, L.X., Yang, Y.H. (2016). Dynamic modeling and contact analysis of a cycloid-pin gear mechanism with a turning arm cylindrical roller bearing. Mechanism and Machine Theory, vol. 104, p. 327-349, D0l:10.1016/j. mechmachtheory.2016.06.018.

[4] Li, T.X., Li, J.B., Deng, X.Z., Yang, J.J., Li, G.G., Ma, W.S. (2017). A new digitized reverse correction method for hypoid gears based on a one-dimensional probe. Measurement Science and Technology, vol. 28, no. 12, art. ID 125004, DOI:10.1088/1361-6501/aa8dd7.

[5] Li, X., Li, C.Y., Wang, Y.W., Chen, B.K., Lim, T.C. (2017). Analysis of a cycloid speed reducer considering tooth profile modification and clearance-fit output mechanism. Journal of Mechanical Design, vol. 139, no. 3, art. ID 033303, DOI:10.1115/1.4035541.

[6] Li, T.X., Tian, M., Xu, H., Deng, X.Z., An, X.T., Su, J.X. (2020). Meshing contact analysis of cycloidal-pin gear in RV reducer considering the influence of manufacturing error. Journal of the Brazilian Society of Mechanical Sciences and Engineering, vol. 42, art. ID 133, D0l:10.1007/s40430-020-2208-7.

[7] Huang, C.H., Tsai, S.J. (2017). A study on loaded tooth contact analysis of a cycloid planetary gear reducer considering friction and bearing roller stiffness. Journal of Advanced Mechanical Design, Systems, and Manufacturing, vol. 11, no. 6, p. 17-00213, Dol:10.1299/jamdsm.2017jamdsm0077.

[8] Demenego, A., Vecchiato, D., Litvin, F.L., Nervegna, N., Manco, S. (2002). Design and simulation of meshing of a cycloidal pump. Mechanism and Machine Theory, vol. 37, no. 3, p. 311332, D0I:10.1016/S0094-114X(01)00074-X.

[9] Hao, D., Fang, Z.D., Hu, Y.H. (2018). Study on the load-sharing characteristics of an aeronautical ii-stage five-branching planets gear train based on the loaded tooth contact analysis. Mathematical Problems in Engineering, vol. 2018, art. ID. 5368294, DOI:10.1155/2018/5368294.

[10] Tsai, S.J., Ye, S.Y. (2018). A computerized approach for loaded tooth contact analysis of planetary gear drives considering relevant deformations. Mechanism and Machine Theory, vol. 122, p. 252-278, D0l:10.1016/j. mechmachtheory.2017.12.026.

[11] Wang, Y.Z., Wu, C.H., Gong, K., Wang, S., Zhao, X.F., Lv, Q.J. (2012). Loaded tooth contact analysis of orthogonal face-gear drives. Proceedings of the Institution of Mechanical Engineers, Part C: Journal of Mechanical Engineering Science, vol. 226, no. 9, p. 2309-2319, DOI:10.1177/0954406211432976.

[12] Bodzás, S. (2020). Computer-aided design and loaded tooth contact analyses of bevel gear pair having straight teeth by 
different loaded torques. Mechanics and Industry, vol. 21, no. 1, art. ID 109, D0I:10.1051/meca/2019076.

[13] Vivet, M., Mundo, D., Tamarozzi, T., Desmet, W. (2018). An analytical model for accurate and numerically efficient tooth contact analysis under load, applied to face-milled spiral bevel gears. Mechanism and Machine Theory, vol. 130, p. 137-156, DOI:10.1016/j.mechmachtheory.2018.08.016.

[14] Lin, C., He, C.J., Gu, S.J., Wu, X.Y. (2017). Loaded tooth contact analysis of curve-face gear pair. Advances in Mechanical Engineering, vol. 9, no. 10, art. ID 1687814017727473, DOI:10.1177/1687814017727473.

[15] Nishino, T. (2009). Computerized modeling and loaded tooth contact analysis of hypoid gears manufactured by face hobbing process. Journal of Advanced Mechanical Design, Systems, and Manufacturing, vol. 3, no. 3, p. 224-235, DOl:10.1299/jamdsm.3.224.

[16] Kolivand, M., Kahraman, A. (2010). An ease-off based method for loaded tooth contact analysis of hypoid gears having local and global surface deviations. Journal of Mechanical Design, vol. 132, no. 7, art. ID 071004, D0I:10.1115/1.4001722.

[17] Batsch, M. (2020). Mathematical model and tooth contact analysis of convexo-concave helical bevel Novikov gear mesh. Mechanism and Machine Theory, vol. 149, art. ID 103842, D0I:10.1016/j.mechmachtheory.2020.103842.

[18] Wang, B., Sun, X., Feng, P.Y., Yan, C.X., Jia, X.J. (2019). Solution and verification of cutter position for machining split equalbase circle bevel gear. Mathematical Problems in Engineering, vol. 2019, art. ID 8024701, D0I:10.1155/2019/8024701.

[19] Yang, J.J., Shi, Z.H., Zhang, H., Li, T.X., Nie, S.W., Wei, B.Y. (2018). Dynamic analysis of spiral bevel and hypoid gears with high order transmission errors. Journal of Sound and Vibration, vol. 417, p. 149-164, D0l:10.1016/j.jsv.2017.12.022.

[20] Changbin, D., Yongping, L., Yongqiao, W. (2020). Dynamic meshing characteristics of elliptic cylinder gear based on tooth contact analysis. International Journal of Engineering, vol. 33, no. 4, p. 676-685, D0l:10.5829/IJE.2020.33.04A.19.

[21] Li, J., Wang, P., Jin, Y. Q., Hu, Q., Chen, X.C. (2016). Cutting force calculation for gear slicing with energy method. International Journal of Advanced Manufacturing Technology, vol. 83, p. 887-896, D0l:10.1007/s00170-015-7630-0.

[22] Wang, Y.R., Ye, H., Jiang, X.H., Tian, A.M. (2018). On the existence of self-excited vibration in thin spur gears: $A$ theoretical model for the estimation of damping by the energy method. Symmetry, vol. 10, no. 12, art. ID 664, D0l:10.3390/ sym10120664.

[23] Wang, J., Feng, F.Z. (2020). Calculation and simulation of time-varying mesh stiffness of involute gear based on energy method. World Scientific Research Journal, vol. 6, no. 1, p. 181-190, D0I:10.6911/WSRJ.202001_6(1).0026.
[24] Liang, X.H., Zuo, M.J., Patel, T.H. (2014). Evaluating the time-varying mesh stiffness of a planetary gear set using the potential energy method. Proceedings of the Institution of Mechanical Engineers, Part C: Journal of Mechanical Engineering Science, vol. 228, no. 3, p. 535-547, DOI:10.1177/0954406213486734.

[25] Wan, Z.G., Cao, H.R., Zi, Y.Y., He, W.P., Chen, Y.M. (2015). Mesh stiffness calculation using an accumulated integral potential energy method and dynamic analysis of helical gears. Mechanism and Machine Theory, vol. 92, p. 447-463, DOI:10.1016/j.mechmachtheory.2015.06.011.

[26] Song, C.S., Zhou, S.W., Zhu, C.C., Yang, X.Y., Li, Z.F., Sun, R.H. (2018). Modeling and analysis of mesh stiffness for straight beveloid gear with parallel axes based on potential energy method. Journal of Advanced Mechanical Design, Systems, and Manufacturing, vol. 12, no. 7, art art. ID JAMDSM0122, DOI:10.1299/jamdsm.2018jamdsm0122.

[27] Wang, J.G., Lv, B., Zhao, Y.X. (2018). Chaos and stability of spur gear transmission system for locomotive based on energy method and floquet theory. Shock and Vibration, vol. 2018, art. ID 5691892, D0I:10.1155/2018/5691892.

[28] Li, T.X., An, X.T., Deng, X.Z., Li, J.F., Li, Y.L. (2020). A new tooth profile modification method of cycloidal gears in precision reducers for robots. Applied Sciences, vol. 10, no. 4, art. ID 1266, Dol:10.3390/app10041266.

[29] Li, T.X., Li, J.F., Deng, X.Z., Tian, M., Li, Y.L. (2020). Quantitative correction method for the grinding errors of cycloidal gears in precision reducer. Journal of Advanced Mechanical Design, Systems, and Manufacturing, vol. 14, no. 4, art. ID JAMDSM0052, D0l:10.1299/Jamdsm.2020jamdsm0052.

[30] Gonzalez-Perez, I., Fuentes-Aznar, A. (2018). Implementation of a finite element model for gear stress analysis based on tie-surface constraints and its validation through the Hertz's theory. Journal of Mechanical Design, vol. 140, no. 2, art. ID 023301, DOI:10.1115/1.4038301.

[31] Xu, H., Shi, Z.Y., Yu, B., Wang, H. (2019). Optimal measurement speed and its determination method in the transmission precision evaluation of precision reducers. Applied Sciences, vol. 9, no. 10, art. ID 2146, D0I:10.3390/app9102146.

[32] Song, M., Wang, H.L., Liu, H.O., Peng, P., Wang, X.H., Pi, D.W., Yang, C., He, G. (2018). Double-layer control of an automatic mechanical transmission clutch during commercial vehicle start-up. Strojniški Vestnik - Journal of Mechanical Engineering, vol. 65, no. 9, p. 515-524, D0l:10.5545/svjme.2019.6144.

[33] Chen, Y.Z., Li, Z., Xie, X.D., Lyu, Y.L. (2018). Design methodology for coplanar axes line gear with controllable sliding rate. Strojniški Vestnik - Journal of Mechanical Engineering, vol. 64, no. 6, p. 362-372, DOI:10.5545/sv-jme.2017.5110. 